\title{
Tecnología radio cognitiva en la banda ultra high frequency (UHF)
}

\section{Cognitive radio technology in the UHF band}

\section{Hernán Paz Penagos}

Ingeniero electrónico, ingeniero electricista, filósofo, magíster en Teleinformática, doctor en Educación. Docente de Escuela Colombiana de Ingeniería Julio Garavito. Bogotá, Colombia. Contacto: hernan.paz@escuelaing.edu.co

\section{MARÍA C. BohórQuez}

Ingeniera electrónica. Analista Mesa de Ayuda del Banco AV Villas. Bogotá, Colombia. Contacto: maria.bohorquez@mail.escuelaing.edu.co

\section{Daniel E. Rodríguez}

Ingeniero electrónico. Consultor junior área GBS-AP de IBM Colombia. Bogotá, Colombia. Contacto:daniel.rodriguez-m@mail.escuelaing.edu.co

\section{Danny O. Maldonado}

Ingeniero electrónico. Ingeniero de Soporte de CRC Outsourcing. Bogotá, Colombia. Contacto: danny.maldonado@mail.escuelaing.edu.co

\section{Claudia L. Sánchez}

Ingeniera electrónica. Investigadora de la Escuela Colombiana de Ingeniería Julio Garavito. Bogotá, Colombia.

Contacto: claudia.sanchez-j@mail.escuelaing.edu.co

Cesar M. Galarza

Ingeniero electrónico, magíster de Ingeniería Electrónica. Asistente de investigación de la Universidad de los Andes. Bogotá, Colombia.

Contacto: cesarmgb@gmail.com

Fecha de recepción: 12 de julio de 2012

Clasificación del artículo: reflexión

Fecha de aceptación: 21 de mayo de 2013

Financiamiento: Escuela Colombiana de Ingeniería Julio Garavito

Palabras clave: banda UHF, comunicaciones celulares móviles, radio cognición, migración.

Keywords: UHF band, mobile cellular communications, cognition radio, migration. 


\section{RESUMEN}

Las empresas de comunicaciones celulares móviles en Colombia requieren mayor recurso espectral para ampliar su portafolio de servicios; sin embargo, no hay frecuencias adicionales para tal fin, no obstante es conocido que existe infrautilización de muchas bandas licenciadas. Por esta razón nuevas tecnologías de radio son estudiadas para su implementación en búsqueda de solucionar este problema, ellas son: Radio Definida por Software (RDS ) [1], Radio Cognición (RC) [2] y Acceso Dinámico al Espectro (ADE) [3]. Estasestrategias proponen movilidad en todo el espectro de las comunicaciones de radio para suplir necesidades y lograr mayor eficiencia en la administración de dicho recurso. Desde esta perspectiva se presenta un estudio de caso para examinar los requisitos que se deben cumplir para la implementación de una red de radio cognitiva en Bogotá, para lo cual se evaluó la posibilidad de migración de las comunicaciones celulares desde sus bandas asignadas a las bandas de televisión UHF, y la coexistencia sin interferencias entre estos 2 servicios. El estudio arrojó viabilidad en dicha migración; sin embargo, la implementación de radio cognitiva compromete voluntades de los operadores y del Ministerio de Tecnologías de la Información y las Comunicaciones de Colombia, y demanda disponibilidad de hardware, software y plataformas de radio flexibles.

\section{ABSTRACT}

Mobile cellular communication companies in Colombia require more spectrum resources to expand their portfolio of services. However, additional frequency bands for that particular purpose are scarce, yet it is well known that there are many underutilized licensed bands. Therefore new radio technologies are being studied in order to solve this problem, e.g. Software Defined Radio SDR [1] Cognitive Radio CR [2] and Dynamic Spectrum Access DSA [3]. These strategies recommend mobility across the radio spectrum to meet various needs and achieve greater efficiency when managing such a scarce resource. In this context, a case study is presented in an attempt to examine the requirements that must be met for the implementation of cognitive radio networks in Bogota. The case study includes evaluation for the possibility of migration from cellular communications to cognitive radio since the bands assigned to UltraHigh Frequency UHF television offer possible free-of-interference coexistence between the two services (i.e. Cellular and TV). The study shows feasibility to migration; however, the implementations of cognitive radio need availability of hardware, software and flexible radio platforms.

\section{INTRODUCCIÓN}

El crecimiento del número de usuarios y de nuevos servicios en las comunicaciones celulares móviles ha generado problemas de saturación de la banda asignada para tal fin según la legislación colombiana; además, un obstáculo que se interpone para su solución radica en que las políticas actuales de asignación de bandas de frecuencia son demasiado rígidas. Sin embargo, hay espe- ranzas de solución, si se considera la aplicación de redes de radio cognitivas que aprovechen de manera oportuna y temporal los huecos espectrales que deja la infrautilización de algunas de esas bandas. Para el desarrollo de este trabajo, la idea inicial fue constatar la necesidad de espectro radio eléctrico, en adelante ER, adicional para las comunicaciones celulares en Bogotá; posteriormente se pasó a verificar la disponibilidad de recurso electromagnético en la banda de fre- 
cuencia ultra-alta (UHF) y finalmente a estudiar los requisitos que se deben cumplir para lograr la migración temporal de las comunicaciones celulares desde su banda asignada hacia los huecos espectrales UHF.

\section{FUNDAMENTOS}

En Colombia el espectro radioeléctrico ha sido definido constitucionalmente como un bien público, cuya gestión y control debe ser realizada por el Estado. Históricamente su planeación y gestión estaban a cargo del Ministerio de Comunicaciones, actualmente Ministerio de Tecnologías de Información y Comunicaciones, cuya denominación y funciones fueron modificadas con la Ley 1341 de 2009. De conformidad con los artículos 75, 101 y 102 de la Constitución Nacional, corresponde al Ministerio de Tecnologías de la Información y las Comunicaciones asignar las frecuencias para la prestación del servicio de telefonía móvil celular, distribuir y definir su cubrimiento; por su parte, a la Comisión de Regulación de Comunicaciones (CRC) le competen algunas funciones de regulación, la Agencia Nacional del Espectro (ANE) es la responsable del monitoreo de todo lo relacionado con espectro para radiodifusión televisiva y la Autoridad Nacional de TV (ANTV) es la responsable del grueso de la parte misional de la Comisión Nacional de TV (CNTV). En este contexto, el espectro electromagnético se divide en bandas de frecuencias fijas por servicios (asignaciones primarias).

Las comunicaciones celulares en Colombia operan en la banda de UHF y las sub-bandas de 850 $\mathrm{MHz}$, bajo la norma GSM (Global System for Mobile Communications) y $1900 \mathrm{MHz}$, bajo la norma UMTS. Los operadores del servicio celular para el primer semestre de 2012 eran los indicados en la tabla 1.

Según estudios realizados por Telefónica y Comcel [4], las empresas de telefonía móvil en Colombia se clasificaban entre las que menos recurso espectral recibían en la región (en otros países se cuenta con anchos de banda desde $50 \mathrm{MHz}$ hasta $80 \mathrm{MHz}$ ); bajo esta premisa, Comcel, Movistar y Tigo buscaron ampliaciones, con lo cual se les asignó $55 \mathrm{MHz}$ del espectro a cada uno, lo que corresponde al "tope del espectro" actual establecido por el Ministerio de Tecnologías de la Información y las Comunicaciones [5] (figura 1).

En este contexto, y de acuerdo a los datos del Ministerio [5] que aparecen en la tabla 2, el número de suscriptores de servicios de telefonía móvil en Colombia sigue incrementándose. El 2005 fue el año de mayor crecimiento, con un incremento del $110 \%$; en ese año Colombia pasó de 10,4 millones a 21,85 millones de abonados. También se observa una disminución de 2,6\% para el 2009 con respecto al 2008 y una ligera recuperación de

Tabla 1. Características de los operadores de telefonía móvil en Colombia

\begin{tabular}{|l|l|l|}
\hline Operador & Sub-banda de frecuencia en MHz & Esquema de acceso \\
\hline Comcel & $\begin{array}{l}\text { Servicios de telefonía móvil sobre una red de tecnología } \\
\text { GSM/GPRS en las bandas de } 850 \text { y1900 }\end{array}$ & TDMA (Time Division Multiple Access) \\
\hline $\begin{array}{l}\text { Movistar: Móviles } \\
\text { Colombia }\end{array}$ & $\begin{array}{l}\text { Servicios de telefonía móvil sobre una red de tecnología } \\
\text { GSM y 3G en las bandas de 850 y 1900 }\end{array}$ & CDMA (Code Division Multiple Access) \\
\hline $\begin{array}{l}\text { Colombia Móvil -Tigo, } \\
\text { antes Colombia Móvil - } \\
\text { OLA }\end{array}$ & $\begin{array}{l}\text { Servicios de telefonía móvil PCS, sobre una red de tecno- } \\
\text { logía GSM/GPRS, EDGE y 3.5G en la banda de los 1900 }\end{array}$ & $\begin{array}{l}\text { TDMA/FDMA (Time Division Multiple } \\
\text { Access/Frequency Division Multiple } \\
\text { Access) }\end{array}$ \\
\hline
\end{tabular}

Fuente: elaboración propia. 


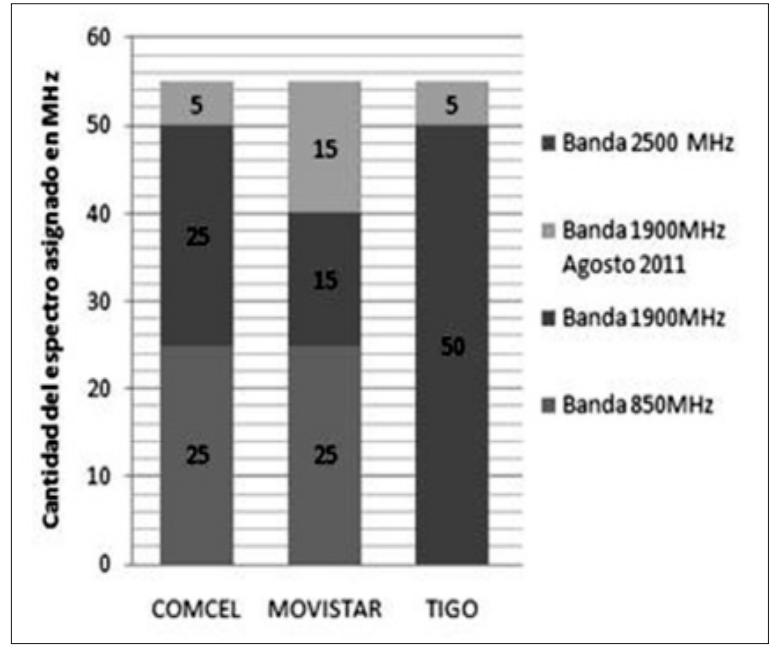

Figura 1. Espectro asignado por International Mobile Telecommunications (IMT) en Colombia

Fuente: adaptada de [21].

6,7\% para el 2010 con respecto del año anterior. Según Cristian Lizcano, director de la CRC, para el primer semestre del 2012 había 46,5 millones de abonados con una penetración superior al $90 \%$.

Con respecto a la distribución del número de abonados por operador, para el primer trimestre de
Tabla 2. Abonados del servicio de telefonía móvil en Colombia

\begin{tabular}{|c|c|}
\hline AÑO & $\begin{array}{c}\text { Abonados en servicio } \\
\text { de telefonía móvil }\end{array}$ \\
\hline 2004 & 10400000 \\
\hline 2005 & 21850000 \\
\hline 2006 & 24912000 \\
\hline 2007 & 33930000 \\
\hline 2008 & 41360000 \\
\hline 2009 & 40300000 \\
\hline 2010 & 43000000 \\
\hline 2011 & 46200000 \\
\hline 2012 & 46500000 \\
\hline
\end{tabular}

Fuente: adaptada de [5].

2009, Colombia móvil mostró una variación del $1,03 \%$, Telefónica móviles del 1,58\% y Comcel del $-1,61 \%$. Asimismo, entre el segundo trimestre de 2009 y el primero del 2010, Colombia móvil mostró una variación del 2,39\%, Telefónica móviles del $-7,93 \%$ pasando de 9,81 millones de abonados a 9,03 millones y Comcel del $0,55 \%$.

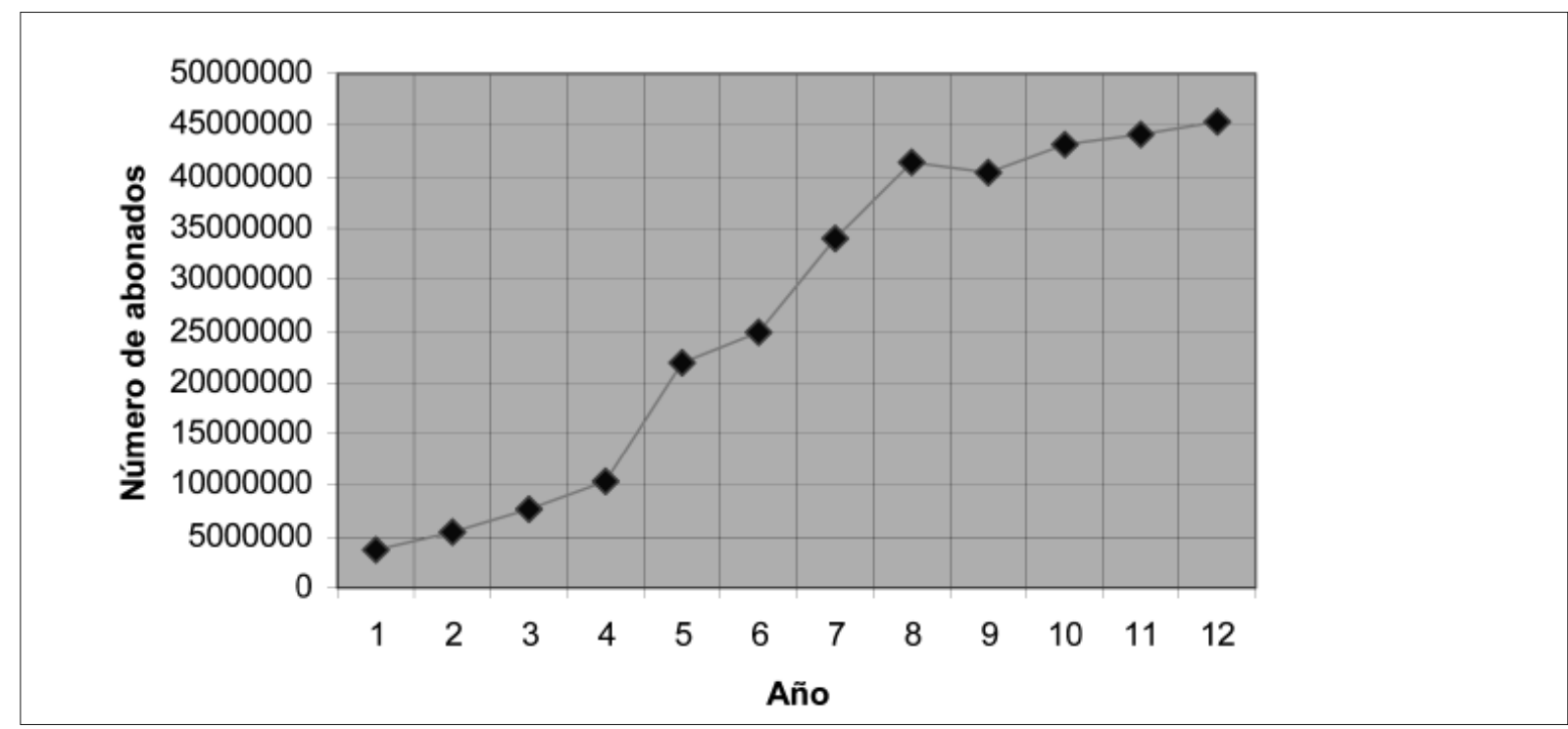

Figura 2. Proyección del número de abonados en servicio de telefonía móvil

Fuente: elaboración propia. 
En resumen, para el primer semestre del 2012 Comcel contaba el $66,49 \%$ de abonados del país, seguido por Movistar que tenía el 21,56\% de participación y Tigo con el 11,95\%.

Desde esta perspectiva y según la figura 2 , se puede inferir que el número de abonados seguirá creciendo; sin embargo, este dependerá de nuevas asignaciones espectrales o de tecnologías que favorezcan el uso eficiente del espectro.

Una política de asignación de espectro fija a servicios específicos, tal y como sucede actualmente en Colombia, puede llegar a infrautilizar el espectro temporalmente, si los usuarios licenciados no dan un uso eficiente del ER. Así lo constatan recientes estudios realizados de manera independiente en diversos puntos geográficos en el mundo [6] - [9]. El uso disperso e ineficiente del ER se presenta de manera particular en los servicios de TV terrestre en Colombia en la banda UHF, tal y como se evidencia en el monitoreo hecho con analizador de espectros (Anritsu $\left.{ }^{\circledR} M S 2721 B 9\right)$ y la antena discono $(A O R \circledast D N 75375)$, durante 24 horas, y que se muestra en la figura 3.

Aunque la figura anterior muestra un bajo nivel de utilización espectral con señales intermitentes de baja y media duración (ráfagas), no proporcionauna idea puntual del grado de infrautilización del espectro, y por consiguiente no es una información suficiente para declarar una banda como realmente disponible para acceso secundario. Sin embargo, esta experiencia es un punto de partida para continuar profundizando en este campo del conocimiento con el ánimo de aportar ideas para mejorar el uso de los recursos espectrales.

Con el advenimiento de la Televisión Digital Terrestre (TDT) a Colombia, se liberarán los canales del 52 al 69 para nuevos servicios de telecomunicaciones y se optimizará el uso del espectro en UHF; en cuanto que, con la misma cantidad de recurso electromagnético utilizado para un canal

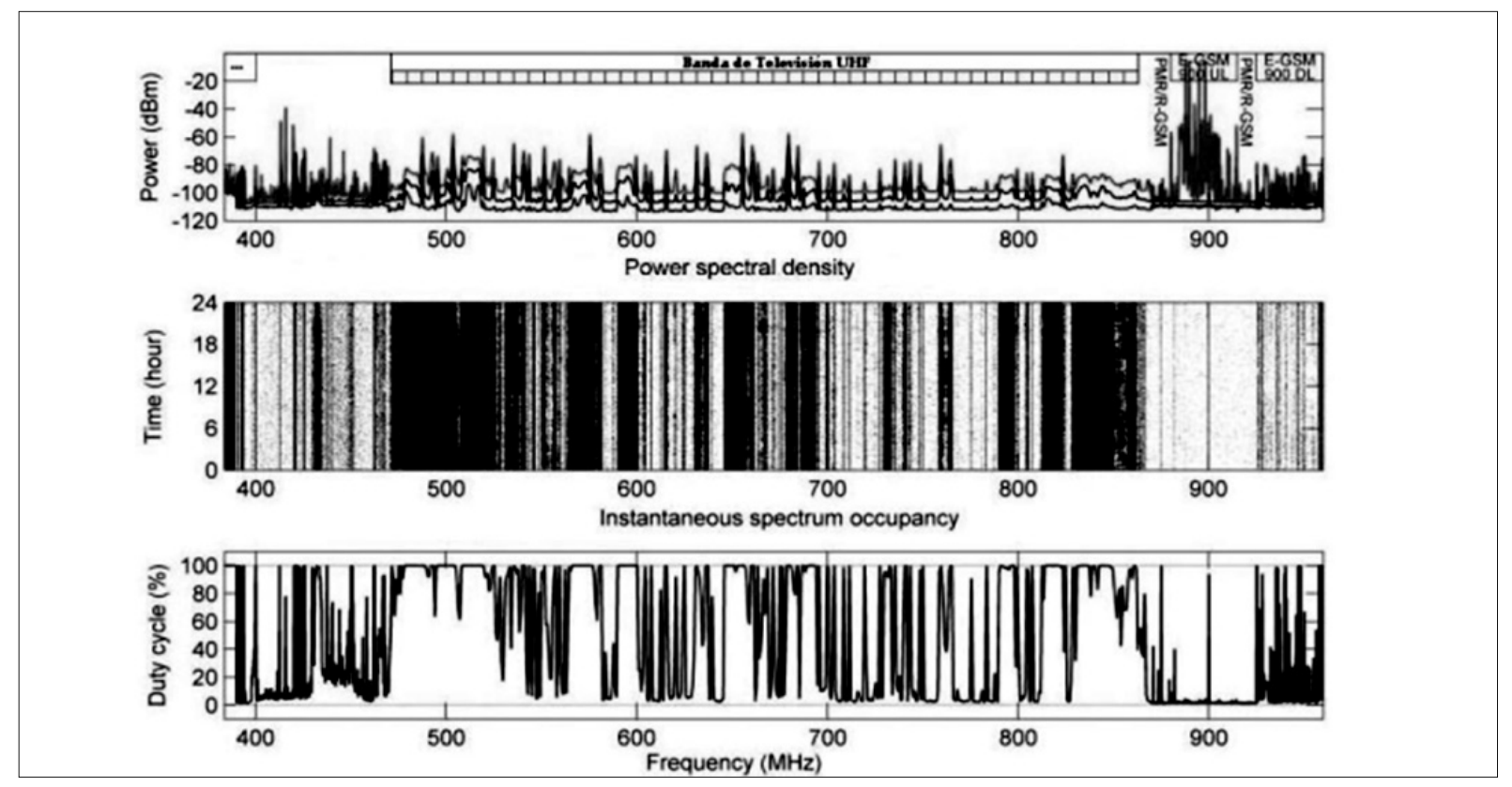

Figura 3. Densidad espectral del servicio de televisión en UHF

Fuente: elaboración propia. 


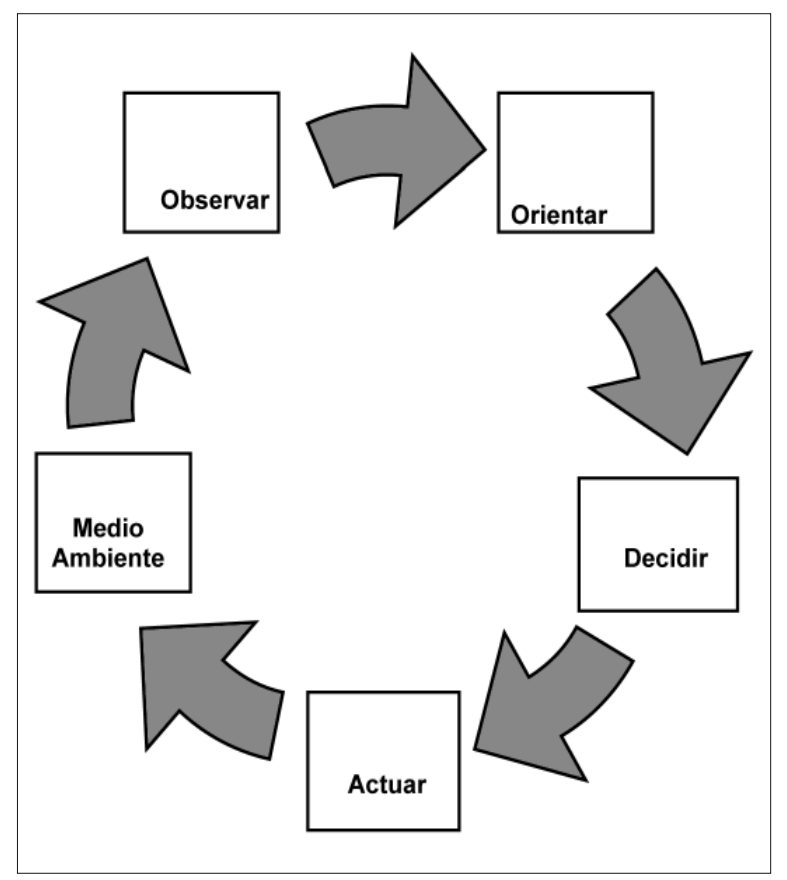

Figura 4. Pasos en el proceso de retroalimentación

Fuente: elaboración propia.

de TV analógico, se podrá transmitir 3 o 4 canales con calidad de imagen de televisión digital estándar (SDTV) o un canal con formato de alta definición (HDTV), esto significará un ahorro de más de $100 \mathrm{MHz}$ en toda la banda de frecuencias. Ahora bien, la transmisión de señal TDT de modo discontinua y en ráfagas, no solo reducirá considerablemente el consumo de potencia media de los terminales, sino que generará silenciamientos en el canal, los cuales se podrán aprovechar para la transmisión de otras señales [10].

Con todo lo anterior, las oportunidades de ampliación de ER mediante la ocupación de bandas libres mediante la introducción de nuevas tecnologías de radio, les da a los operadores la posibilidad de migrar permanente o temporalmente sus nuevos servicios hacia las bandas de frecuencia disponibles, y así descongestionar el tráfico denso que se presenta en las bandas legítimamente otorgadas para el segmento de comunicaciones móviles celulares.

\section{RADIO COGNITIVA}

Se define como la tecnología que cambia sus parámetros de radio-transmisión basándose en la interacción con el medio. La idea básica consiste en permitir que usuarios secundarios puedan acceder de manera oportuna a ciertas bandas del espectro temporalmente desocupadas, siempre y cuando dichas transmisiones no interfieran en las transmisiones de los usuarios primarios [11].

Se fundamenta en la retroalimentación en bucle de un proceso que tiene en el medio ambiente su punto de partida y llegada (figura 4), y está constituida por 4 unidades fundamentales: 1) una unidad de sensado que observa las comunicaciones ya establecidas y por establecerse en el nodo de la red, y proporciona información respecto al entorno, en términos, por ejemplo, de ocupación del espectro; 2) un mecanismo cognitivo que estructura la información sensada, que puede tener conocimiento de criterios complementarios como, por ejemplo, políticas de gestión del espectro, máscaras de espectro, etc., y a partir de dicha información orientar el tráfico emergente; 3) una unidad de decisión que determina cuándo y cómo la radio debe reconfigurarse para adaptarse a los cambios del entorno; y 4) una unidad de radio flexible que modifica los parámetros de transmisión y recepción con base en las decisiones tomadas por la unidad de decisión.

Las funciones principales de la RC son: detectar, administrar, moverse y compartir el espectro; un ejemplo de este tipo de radio es la especificación IEEE 802.15 del grupo 2 [12].

De acuerdo con lo anterior, la tecnología RC permitirá a los usuarios: (1) determinar qué porciones del espectro están disponibles y detectar la presencia de los usuarios con licencia cuando un usuario secundario esté operando en dicha banda; la manera de lograrlo mediante las siguientes estrategias: a) detección de transmisiones de 
usuarios primarios (legítimos), b) detección cooperativa: en este caso diferentes usuarios RC intercambian periódicamente información sobre la detección de usuarios primarios y c) detección basada en interferencias. 2) Seleccionar el mejor canal disponible (gestión de ER), 3) coordinar el acceso a los canales disponibles con otros usuarios secundarios (compartir el ER), y 4) abandonar el canal cuando se detecte un usuario primario (movilidad de ER).

El IEEE (Institute of Electrical and Electronics Engineers) creó en abril de 2007 el SCC41 (Standards Coordinating Committee 41), con el objetivo de desarrollar estándares para RC [12]. Este comité está dividido en grupos de trabajo y grupos de estudio, los cuales se identifican como IEEE P1900.x. Asimismo, diferentes asociaciones internacionales sin ánimo de lucro también trabajan en el ámbito de la RC. La más representativa es el SDR Forum. De manera complementaria existe la $\operatorname{CogNeA}$ (Cognitive Network Alliance) y la ECMA (European Computer Manufacturers Association) que a menudo contribuyen con los órganos de estandarización, como ETSI o ISO (International Organization for Standarization).

La tecnología $\mathrm{RC}$ existe gracias a los avances en recursos informáticos, nuevas estrategias de conocimiento, software de comunicaciones y nuevos dispositivos electrónicos para el monitoreo del ER y asignación de recursos de radio en tiempo real [13], [14]; actualmente utiliza la RDS, la radio adaptativa, y técnicas disruptivas tales como senseo del espectro.

Uno de los principales inconvenientes que presentan algunas aplicaciones RC es la limitación técnica (señalización del canal, sensitividad, barrido del espectro, autoabastecimiento de energía) de los sensores electrónicos que se ocupan de detectar correctamente la presencia de señales en el espectro (estado de la red); por tanto, se estudian nuevas estrategias multiportadora
COFDM/adaptativa (Coded Orthogonal Frequency - Division Multiplexing), para superar las limitaciones antes mencionadas, o reforzar la función de los sensores para garantizar una detección y señalización confiables, y una migración sin interferencias [15].

Para el estudio de caso en consideración se propone para la transmisión RC un esquema multiusuario COFDM simple, operando en el modo de $8 \mathrm{~K}$ (6817 portadoras) de la norma europea EN 300744 [16], esto debido a su recomendación para escenarios con un elevado índice de señal multitrayecto como lo son los ambientes urbano y suburbano de Bogotá.

\section{ESTUDIO DE CASO: MIGRACIÓN DE LAS COMUNICACIONES CELULARES A LA BANDA DE TELEVISIÓN UHF EN BOGOTÁ (COLOMBIA)}

Ya se han desarrollado trabajos en este sentido [17], en los cuales se utiliza la RC para facilitar la migración de tecnologías WRAN a espectros asignados y no utilizados para la TV. Otro caso se puede consultar bajo el estándar IEEE802.22 WG, en calidad de borrador [18]. También está en desarrollo el proyecto 802.16h, de WiMAX (World wide Interoperability for Microwave Access), el cual incorpora a su red mecanismos para mejorar la coexistencia de operaciones licenciadas [9]. Estas y otras pruebas de campo han demostrado que es posible la migración de comunicaciones celulares desde las bandas licenciadas a la banda IV-V de televisión, si se adopta una correcta configuración de los parámetros de transmisión de la red y si se incorporan algunas innovaciones en el nivel de aplicación compatibles con el estándar. A continuación se revisarán algunas de ellas:

- Para pasar de un núcleo de red centralizada hacia otra distribuida, es importantedesagregar geográficamente la demanda, cam- 
biar las funcionalidades de los equipos de red núcleo, introducir localizaciones de diseño para los equipos de red de núcleo y cambiar la tecnología de la infraestructura de la transmisión arrendada.

- Contar con una interfaz de radio fija punto a multipunto, a través de la cual la estación base EB controle la celda y todos los equipos locales de usuarios (Consumer Premise Equipments: CPE).

- Cada CPE debe contar con el servicio de una antena inteligente o de arreglos de antenas (puede ser del tipo panel UHF, con ganancias superiores o iguales a $13,37 \mathrm{~dB}$ ), que le permitan a la EB comunicarse con otras EB y monitorear la disponibilidad del espectro.

- La interfaz aérea, en sus premisas de subida y bajada, debe contar con una capa física y Media Access Control (MAC) robustas que detecten ER disponible; asimismo, procesadores de señal flexibles que garanticen la migración sin interferencias de las comunicaciones secundarias a dichas franjas (coexistencia y autocoexistencia).
- La EB se encarga de controlar el acceso a la celda y transmitir información de control a varios CPE; además, administrará la percepción distribuida, característica necesaria para asegurar el correcto uso del espectro de televisión, sin interferir a los usuarios licenciados.

- Según el tipo de celda (macro, micro, pico) se debe emitir una potencia que garantice la cobertura; sin embargo, esta no debe interferir en las comunicaciones de otros usuarios. Por ejemplo, una estación base con una potencia radiada efectiva entre 10 a $50 \mathrm{Wen}$ GSM, puede cubrir en promedio $33 \mathrm{~km}, \mathrm{y}$ también causar interferencias.

- Contar con una radio configurable por software (de la capa física), que a mediante el monitoreo del entorno, identifique las comunicaciones en curso y sus cambios, y como respuesta al nuevo estado de la red, disponga, en tiempo real, los recursos necesarios.

Como se evidencia en la siguiente figura, las oportunidades de transmisión de las EB son totalmente aleatorias. Esta característica se debe tener

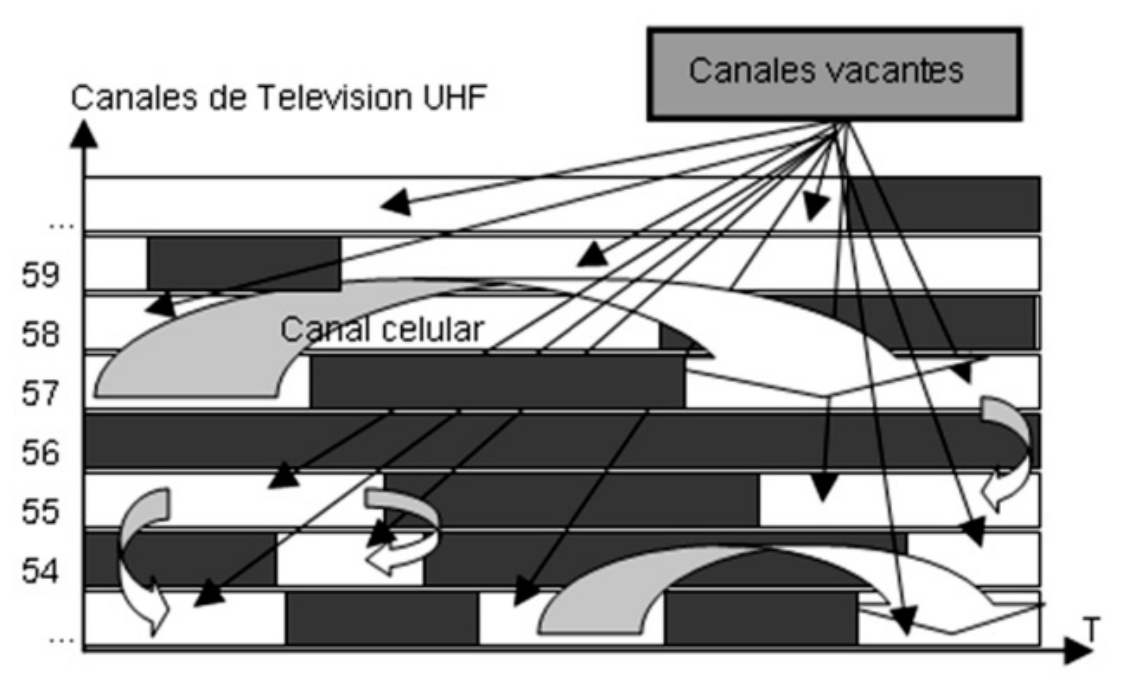

Figura 5. Acceso dinámico al espectro

Fuente: elaboración propia. 
en cuenta en el diseño de la capa MAC y del nivel físico.

Para sincronizar la red e informar a los servidores centralizados sobre la disponibilidad del espectro local, cada EB debe contar con un receptor GPS (Global Positioning System). El centro de conmutación móvil (CCM) de la celda les informará a los nodos de la RC, los canales de TV libre y las bandas de guarda susceptibles a utilizar. Ahora bien, si los canales de TV están transmitiendo señal, se recurriría al uso del mecanismo de fase de senseo para monitorear el estado del canal durante intervalos de tiempo aleatorio, para lo cual se emplea un algoritmo de senseo rápido, que le puede tomar algunos milisegundos por canal. Los resultados de las medidas de los CPE se centralizan en las EB, las cuales toman decisiones dependiendo de la respuesta [13].

Una de las funciones de las antenas en las EB y los CPE es identificar la presencia de emisiones de los usuarios primarios. Las EB abandonan un canal si detectan señales de TV digital o analógica con umbrales de $-116 \mathrm{~dB}$ para un canal de $6 \mathrm{MHz}$.
Como lo muestra la figura 6, el plan de frecuencias del servicio de TV terrestre UHF en Colombia cuenta con 48 canales ubicados en la banda IV-V de 512-806 MHz, así: 512 a $798 \mathrm{MHz}$ (canales 21-51) y 798 a $806 \mathrm{MHz}$ (canales 52-69).

La capa física debe conocer previamente el comportamiento del canal de radio en cuanto al tipo de desvanecimiento y efectos multitrayectoria de la señal en las subandas por utilizar. Una estrategia para identificar el comportamiento del canal de radio es monitorear su estado en tiempo real; para lograr esto, se sugiere utilizar subportadoras pilotos COFDM con el propósito no solo de identificar los huecos espectrales propicios para la transmisión de comunicaciones celulares, sino para estimar el estado del canal.

La recuperación de una señal de prueba transmitida en la EB, dará cuenta de 2 parámetros clave: información sobre el estado del canal y la tasa de error de bit. El primero es un parámetro global sobre el comportamiento del canal digital, el cual no podrá estar por debajo de un límite determinado (por ejemplo $\pm 50 \%$ ); y el segundo informa

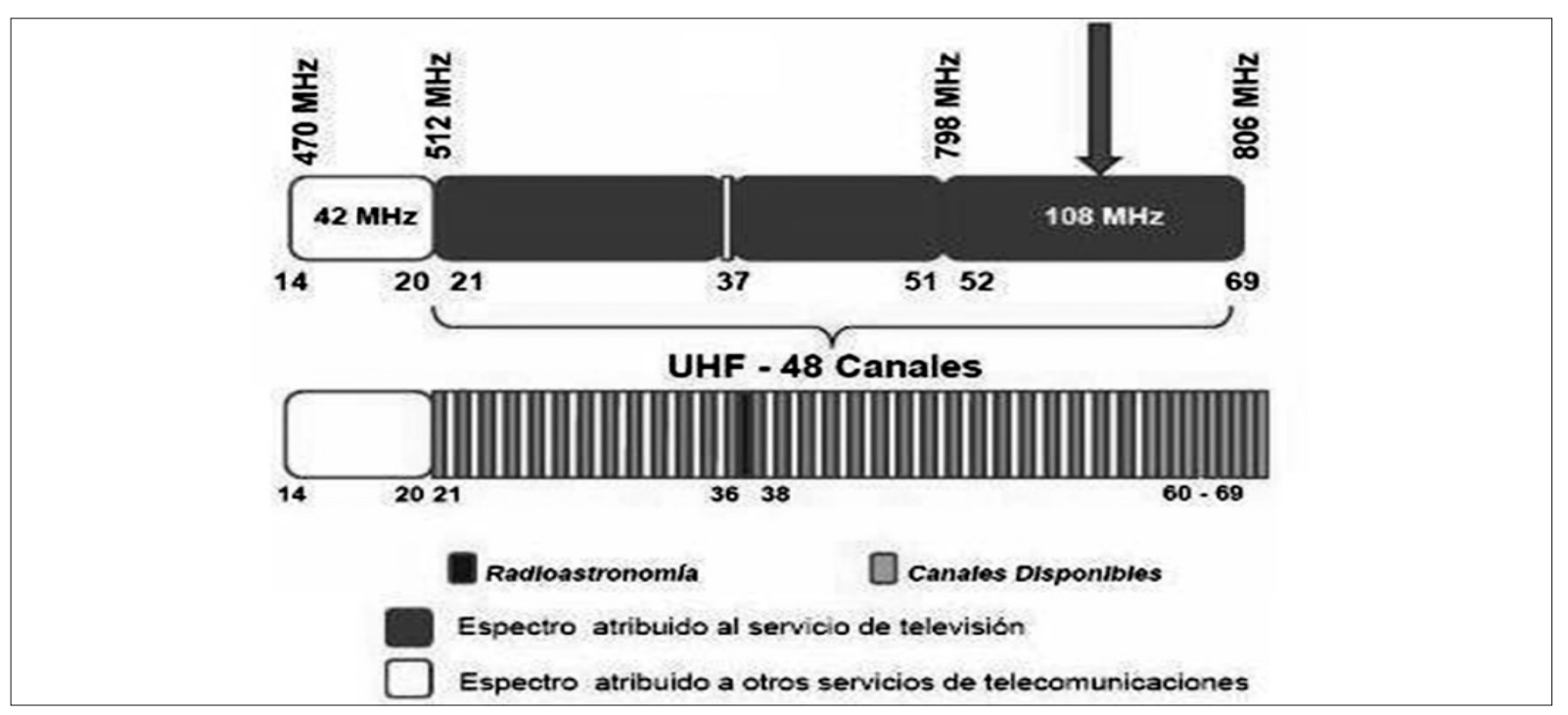

Figura 6. Ancho de banda del canal de televisión analógico

Fuente: elaboración propia. 
sobre el número de errores en la señal recibida, tomando en cuenta que el umbral establecido para el QEF (Quasi Error Free) en sistemas digitales es de $2 \times 10^{-4}$. Con los valores adecuados en estos parámetros se conseguirá buena QoS. Un aspecto crítico para considerar en la planeación de la RC es el tiempo de propagación de la señal COFDM. Para el modo 8K, sugerido para este caso de estudio, se debe usar un prefijo cíclico de $28 \mu \mathrm{s}$; es decir, de $1 / 32 T_{s}$, para reducir la sobrecarga. Sin embargo, ese retardo puede ser insignificante frente al intervalo de guarda tolerable de $224 \mu$ s en redes de TV SFN (Single Frecuency Networks).

El papel clave de la RC es el control de la interferencia en el receptor primario, que se convierte en un problema sin fácil solución, en el escenario que nos ocupa, dado que dicho receptor (TV) es pasivo. Para garantizar las comunicaciones sin interferencias de los usuarios primarios, y al mismo tiempo permitir comunicaciones de los usuarios secundarios (abonado celular), es pertinente utilizar técnicas de transmisión cooperativa entre usuarios secundarios, lo que permitiría maximizar la tasa de transmisión con niveles de potencia bajos y sin causar interferencias. De forma similar, se podría aplicar los conceptos de cooperación entre nodos en entornos cognitivos, para que los usuarios secundarios compensen la interferencia causada a los receptores pasivos de los usuarios primarios. Esta cooperación se hace mediante reenvío al receptor primario de mensajes primarios interceptados por los transmisores secundarios debido a las características de la radiodifusión en un entorno inalámbrico. El receptor primario (usuario de TV) podrá así mejorar la robustez de su proceso de detección, gracias a la redundancia de los mensajes recibidos y a su diversidad.

Asimismo, se podrían controlar parte de las interferencias insertando intervalos de guarda entre las subportadoras OFDM que modulan los datos; sin embargo, esta estrategia reduce la capacidad del canal cuanto mayor sea el número de porta- doras utilizadas. La tabla 3 especifica el intervalo de guarda para TV digital y compara otras características de dicha TV con la TV analógica que coexiste hoy en Colombia en esa transición del apagón analógico.

Siempre que una CPE se encienda, lo primero que se debe hacer es escanear todos los canales de RC, después reconstruir un mapa de la ocupación espectral, en el cual se identifica presencia de usuarios primaros y secundarios en la banda UHF. Esta información es enviada a la EB y usada por el CCM para determinar por cuales canales vacantes se realizarán transmisiones $\mathrm{SCH}$ (Superframe Control Header) de las EB. Una vez que la $\mathrm{CPE}$ recibe el $\mathrm{SCH}$, toma el canal y accede a la red. Las mediciones aleatorias que informan sobre el grado de ocupación del ER, pueden ser de dos tipos: 1) medidas en banda: que se realizan con los canales usados por la EB para comunicarse con las CPE. Para estas medidas la EB silencia periódicamente el canal, así el detector (antena) de usuarios primarios puede cumplir su cometido, o 2) medidas fuera de banda: en este caso las medidas se realizan con los canales restantes. Para detectar la presencia de usuarios primarios, las antenas del CPE y la EB deben contar con una sensitividad suficiente para detectar con acierto señales con relación señal a ruido muy bajo. Otra alternativa para lograr la coexistencia entre los usuarios de TV y abonados celulares sin interferencia, es utilizar una red de sensores auxiliares que recojan la información sobre ocupación espectral de los usuarios primarios e informen a las EB para habilitar transmisiones de los usuarios secundarios. Esta información se podría, por ejemplo, proporcionar a unabase de datos a la cual los secundarios accederían antes de realizar transmisiones en banda licenciada.

El surgimiento de un mercado secundario del espectro conlleva necesariamente el desarrollo de sistemas de información en tiempo real sobre el espectro utilizado (frecuencias utilizadas 
Tabla 3. Especificaciones de los parámetros de las señales de TV analógica y digital que coexisten en el proceso de apagón analógico en Colombia

\begin{tabular}{|c|c|c|}
\hline \multirow{2}{*}{ Parámetro } & \multicolumn{2}{|c|}{ Señal de televisión } \\
\hline & Analógica & Digital \\
\hline Ancho de banda & $\begin{array}{l}6 \mathrm{MHz}+0,25 \mathrm{MHz} \text { de banda de guarda. } \\
\text { Las bandas de guarda adyacentes al ca- } \\
\text { nal emisor han de estar libres para evitar } \\
\text { interferencias. }\end{array}$ & $\begin{array}{l}\text { Originalmente diseñado para canales de } 8 \mathrm{MHz} \text {; sin embar- } \\
\text { go, se aplicará para el caso colombiano de } 6 \mathrm{MHz}\end{array}$ \\
\hline Estándar & $\begin{array}{l}\text { NTSC-M (National Television System Com- } \\
\text { mittee), EstadosUnidos. }\end{array}$ & $\begin{array}{l}\text { DVB-T2: Digital Video Broadcasting - Terrestrial-segunda } \\
\text { generación. }\end{array}$ \\
\hline Codificación & $\begin{array}{l}\text { La televisión normal analógica se transmite } \\
\text { con resolución } 480 \mathrm{i} \text {, en } 525 \text { líneas vertical, } \\
30 \text { cuadros por segundo y } 60 \mathrm{~Hz} \text {. }\end{array}$ & $\begin{array}{l}\text { Se codifican en tramas de datos PES (Packetized Elemen- } \\
\text { tary Stream) y se transmiten por paquetes de } 188 \text { bytes }\end{array}$ \\
\hline Tipo de servicios & Audio y video & Video, audio y datos (teletexto y subtítulos) \\
\hline Nivel de señal & $\begin{array}{l}57-80 \mathrm{~dB} \text { o equivalente a } 60 \mathrm{~dB} \mu \mathrm{V} / \mathrm{m} \text { de in- } \\
\text { tensidad de campo en el receptor. }\end{array}$ & $\begin{array}{l}\text { 47-70dB. Una recepción digital óptima necesita menor po- } \\
\text { tencia de señal que una equivalente analógica. }\end{array}$ \\
\hline $\begin{array}{l}\text { Técnicas } \\
\text { de modulación }\end{array}$ & $\begin{array}{l}\text { Video: AM en banda lateral vestigial. } \\
\text { Color: QAM. } \\
\text { Audio: FM }\end{array}$ & $\begin{array}{l}\text { 64-QAM / COFDM con } 1 \mathrm{~K}, 2 \mathrm{~K}, 4 \mathrm{~K}, 8 \mathrm{~K}, 8 \mathrm{~K} \text { ext., } 16 \mathrm{~K}, 16 \mathrm{~K} \\
\text { ext., 32K y } 32 \mathrm{~K} \text { ext.portadoras, con intervalo de guarda } \\
\text { de } 1 / 4,1 / 8,1 / 16,1 / 32,1 / 128,19 / 128 \text { y } 19 / 256 \text {. } \\
\text { Estrategias: constelaciones rotadas, entrelazado temporal } \\
\text { y transmisión con diversidad espacial (MISO) }\end{array}$ \\
\hline Procesamiento & $\begin{array}{l}\text { Multiplexación de canales de video, audio, } \\
\text { color y sincronización }\end{array}$ & $\begin{array}{l}\text { Compresión: MPEG-4: (Parte 10) y H.264. Corrección de } \\
\text { error: FEC: } 1 / 2,3 / 5,2 / 3,3 / 4,4 / 5,5 / 6 \text { y técnicas mejoradas } \\
\text { LDPC+BCH. }\end{array}$ \\
\hline
\end{tabular}

Fuente: adaptada de [22] y [23].

y disponibles, modulaciones empleadas, ámbito espacial de aplicación, etc.), recursos disponibles para los terminales de usuario (frecuencias de trabajo, modulaciones, tecnologías compatibles, tasas de transferencia, retardos y latencias en función de la banda y tecnología usada, geolocalización, etc.), así como el modelado de las estaciones base desplegadas y la caracterización de los servicios por ofrecer (tasas de transferencia necesarias, tolerancia a retardos e interrupciones de sesión, necesidades de autenticación, etc.).

Para lo anterior, las nuevas técnicas y métodos de Asignación Dinámica del Espectro (ADE) necesitarán de información compartida, gestión y coordinación tanto de los operadores como de las tecnologías inalámbricas aplicadas. Además es importante tener en cuenta el tiempo de transición que llevará la Televisión Digital Terrestre
(TDT) en Colombia, tiempo en el cual convivirán 2 servicios de difusión de televisión y en el que no es posible una planificación técnica definitiva de la RC.

Por otra parte, los nuevos algoritmos de procesado de la señal, basados en detectores de energía o en propiedades ciclo estacionarias, son capaces de encontrar un compromiso entre la fiabilidad del sensado y el nivel de conocimiento que el sistema secundario debe tener del sistema primario. Por este motivo, es necesario desarrollar plataformas de radio que sean capaces de soportar la implementación de dichos algoritmos. Asimismo, es fundamental integrar nuevas arquitecturas que permitan realizar el sensado de múltiples canales en paralelo sin perder flexibilidad en lo referente a su capacidad de modulación de la frecuencia central [19], [20]. 
Por otra parte, un mejor uso del espectro radioeléctrico y significativos ahorros de infraestructura y energía ahora son posibles por medio del diseño e implementación de redes DVB-T2 SFN de gran tamaño. Topologías de red con separación entre transmisores de hasta 2,5 veces más (212 km @ $6 \mathrm{MHz}$ ) la separación permitida en DVB-T. De igual manera, y como lo hará la DVB-T2, otros sistemas de radio digital podrán ofrecer mayor protección frente a interferencias para transmisiones en el mismo canal RF o en canales adyacentes, gracias a la utilización de procesados Fast Fourier Transform (FFT) o Wavelet de la información. Esto significa, por ejemplo para el caso de Colombia, poder instalar pocos transmisores en la ciudad de Bogotá emitiendo en una única frecuencia, sin riesgo a interferir el rendimiento de las estaciones adyacentes y por el contrario, contribuyendo positivamente a la potencia total presente en la antena del receptor.

\section{CONCLUSIONES}

Para que las comunicaciones móviles celulares puedan beneficiarse del concepto de radio cognitiva en Bogotá, es necesario disponer de plataformas radio flexibles. Sin embargo, las plataformas de radio disponibles no resultan adecuadas en términos de flexibilidad, costo y gasto energético, por ejemplo, para realizar de manera eficiente labores de escaneado de frecuencias y detección de bandas ocupadas y libres.
El desarrollo de soluciones prácticas requiere estudios de ocupación espectral en escenarios realistas que proporcionen la información necesaria para que las soluciones desarrolladas den una respuesta satisfactoria a dichas situaciones planteadas. En concreto se sugiere estudiar entornos de aplicación en busca de escenarios que:1) abarquen bandas de frecuencias en las cuales la densidad de uso del espectro, tanto libre como licenciado, sea relevante para el estudio de viabilidad de la solución propuesta; 2) presenten una utilización significativa y habitual de sistemas en bandas licenciadas con terminales tanto activos (telefonía móvil, etc.) como pasivos (televisión, radio, etc.); 3 ) permitan el estudio en condiciones tanto estáticas como en movilidad; y 4) comprendan toda la cadena de valor en la aplicabilidad de la tecnología, desde el usuario final hasta el proveedor último de servicios sobre ella.

Los sistemas cognitivos constituyen una tecnología extremadamente prometedora, pero su eventual desarrollo se ve comprometido por problemas de implementación, tanto desde el punto de vista hardware, como software, así como por la disponibilidad de plataformas de radios flexibles y simuladores. La implementación de RC en las redes celulares de Bogotá implica ajustes importantes en los CCM, CPE, las EB y el sistema de antenas para radio cognición que implican características inteligentes. Además, si se proyecta una red radio cognitiva en Bogotá se podrían reutilizar los emplazamientos celulares y de televisión construidos (torres de televisión y radio).

\section{REFERENCIAS}

[1] J. Kim, S.Hyeon, andS. Choi, Implementation of an SDR system using graphics processing Unit., IEEE communications magazine, pp. 156-162, March,2010.

[2] E. Nicollet, Standardizing transceiver APIs for software defined and cognitive radio,
RF design magazine, pp. 16-20, February, 2008.

[3] V.R. Petty, R. Rajbanshi, D. Datla, F. Weidling, D. De Pardo, and P. J. Kolodzy,; M. J. Marcus, A. M. Wyglinski, J.B. Evans, G. J. Mindenand J. A. Roberts, Feasibility 
of Dynamic Spectrum Access in Underutilized Television Bands, 2nd IEEE International Symposium on New Frontiers in Dynamic Spectrum Access Networks (DySPAN 2007), pp. 331-339, April, 2007.

[4] UIT: Asignación, gestión y aplicación del espectro radio eléctrico. En línea. Disponible en: http://www.tendencias21.net/ La-UIT-actualiza-el-tratado-internacionalque-rige-las-frecuencias-radioelectricas a1947.html.

[5] TIC: Ministerio de Tecnologías de la Información y las Comunicaciones, "Cifras primer trimestre 2012. República de Colombia, mayo del 2012", Boletín trimestral de las TIC. Banda ancha* Vive digital. En línea. Disponible en http://www. mintic.gov.co/images/documentos/cifras del_sector/boletin_banda_ancha_vive_ digital_1t_2012.pdf.

[6] Shared Spectrum Company, Spectrum occupancy measurements, 2010. En línea. Disponible en http://www.sharedspectrum. com/measurements.

[7] R. Chiang, G. Roweand K. Sowerby, Aquantitative analysis of spectral occupancy measurements for cognitive radio., IEEE 65th Vehicular Technology Conference, VTC 2007-Spring, pp. 3016-3020, April, 2007.

[8] M. Wellens, J. Wu, and P. Mähönen, Evaluation of spectrum occupancy in indoor and outdoor scenario in the context of cognitive radio. Second International Conference on Cognitive Radio Oriented Wireless Networks and Communications, August, 2007, p. 8.

[9] M.H. Islam, et ál., Spectrum survey in Singapore: Occupancy measurements and analyses, 3rd International Conference on
Cognitive Radio Oriented Wireless Networks and Communications (CrownCom 2008), May, 2008, p. 7.

[10] M. López-Benítez, F.Casadevall, A. Umbert, J. Perez-Romero, R. Hachemani, J. Palicot, and C. Moy, Spectral occupation measurements and blind standard recognition sensor for cognitive radio networks, 4th International Conference on Cognitive Radio Oriented Wireless Networks and Communications (CrownCom 2009), June, 2009, p. 9.

[11] F. Capar, et ál., Analysis of coexistence strategies for cellular and wireless local area networks. VTC Fall 2003, October, 2003.

[12] IEEE Standards in communications and networking, Architecture and Enablers for Optimized Radio Resource Usage in Heterogeneous Wireless Access Networks, The IEEE 1900. 4 Working Group.

[13] A. Timo, "Spectrum pooling: An innovative strategy for the enhancement of spectrum efficiency", IEEE Radio Communications, March, 2004.

[14] S. Wolfram, Cellular Automata and Complexity: Collected Papers, 2009.

[15] P. Leaves, et ál., Dynamic spectrum allocation in composite reconfigurable wireless networks, IEEE Communications Magazine, May, 2004. En línea. Disponible en http://www.ieeep1900.org/.

[16] D. Gómez-Barquero, A.Bria, J. F. Monserratand N. Cardona, Minimal Cost Planning of DVB-H Networks on Existing Wireless Infrastructure, Proceeding IEEE Personal, Indoor and Mobile Radio Communications (PIMRC), Helsinki, Finland, 2006. 
[17] ETSI European Standard. Digital Video Broadcasting (DVB), Second generation framing structure, channel coding and modulation systems for Broadcasting, Interactive Services, News Gathering and other broadband satellite applications (DVB-S2) EN 302307 V1.2.1 (2009-08)

[18] C. Cordeiro, K.Challapali, D. Birruand S. Shankar, IEEE 802.22: the first worldwide wireless standard based on cognitive radios, in: Proc. IEEE D y SPAN 2005, November, 2005, pp. 328-337.

[19] H. Poor, An Introduction to Signal Detection and Estimation, New York: SpringerVerlag, 1985, chapter 4. En línea. Disponible en http://es.wikipedia.org/wiki/ Spectrum_Pooling.
[20] M. Wellens, et ál., Exploiting Historical Spectrum Occupancy Information for Adaptive Spectrum Sensing, Department of Wireless Networks, RWTH Aachen University, 2009.

[21] Agencia nacional del espectro. En línea. Disponible en www.ane.gov.co/apc-aafiles/ $35383137643637613966333438336638 /$ Presentaci_n190015abril.pdf

[22] Informe anual de la televisión. En línea. Disponible en: 2012www.cntv.org.co

[23] ACIEM. Parámetros técnicos para DVBT2. Revista de la Asociación Colombiana de Ingenieros: ACIEM. Edición No.114. enero-febrero-marzo 2012. 